\title{
Influence of relevant parameters on the extraction efficiency and the stability of the microdrop in the single drop microextraction
}

\author{
Silvia Zichová, Adriána Brisudová, Svetlana Hrouzková \\ Slovak University of Technology in Bratislava, Faculty of Chemical and Food Technology, \\ Institute of Analytical Chemistry, Radlinského 9, 81237 Bratislava, Slovak Republic \\ svetlana.hrouzkova@stuba.sk
}

\begin{abstract}
Single drop microextraction technique uses microamounts of organic solvents. Simplicity, low cost, low environmental impact, compatibility with chromatographic systems as well as its applicability to different matrices are main advantages of single drop microextraction. This technique has become frequently used for the extraction of a broad scope of compounds for numerous analytical applications. This review provides an overview of the existing single drop microextraction modes of realisation and the main scope is devoted to the optimization of parameters influencing the efficiency. The state of the art is discussed on the basis of examples selected from representative application areas. Extraction parameters for toxic organic compounds extraction and microdrop stability were evaluated.
\end{abstract}

Key words: single drop microextraction, drop stability, toxic organic compounds, extraction efficiency

\section{Introduction}

In the frame of analytical methods, sample preparation is considered to be the potentially most limiting step of the whole procedure, since it is multistep process which usually requires the use of organic solvents (Tobiszewski et al., 2009; Andruch et al., 2012). The proper selection of a sample preparation methodology is a crucial point, which is highly dependent on both, analyte and sample nature. Although various conventional commonly used sample preparation techniques exist, they all possess drawbacks, such as poor operability, labor-intensive procedures, time consuming, complicated, expensive, require large amounts of organic solvents which result in the production of hazardous laboratory waste and usually involve many steps, leading to loss of some analyte quantity (Hrouzková, 2017). Modern trends in sample preparation are towards the simplification and miniaturization, bringing especially minimalization of the volume of organic solvent used (Hrouzková et al., 2018). A variety of techniques based on solvent-extraction principle employing the minimal volume of organic solvent is generally named liquid phase microextraction (LPME) techniques. LPME have been recently reviewed by Asensio-Ramos et al., 2011, SarafrazYazdi and Amiri, 2010, Andrašč́ková et al., 2015, $\mathrm{Xu}$ et al., 2007. LPME has been elaborated under various approaches, one of those approaches was termed single-drop microextraction (SDME).
SDME is based on the distribution of analytes between a microdrop of extraction solvent of microliters volumes at the tip of a microsyringe needle and a liquid sample containing the analytes or in the headspace area above the liquid/solid sample. The syringe needle is used to pierce the septum of a closed container. When the tip of the needle is in the desired position (in the aqueous phase or in the headspace), a hanging droplet of solvent is exposed to the matrix by depressing the plunger of the syringe. After extraction is completed, the droplet is withdrawn into the syringe barrel by lifting the plunger and injected into separation systems for the subsequent analysis. SDME was applied in a wide range of applications, involving different arrangements.

One of the aims of this paper is to overview the existing SDME modes of realization with respect to the experimental arrangement. Development of a particular analytical procedure for the determination of analytes of toxic organic compounds by SDME requires optimization of various parameters related to the extraction step. The important aim of this paper is devoted to overview the variables and experimental parameters, such as the extraction solvent selection and its volume, agitation, temperature, $\mathrm{pH}$ of the sample and the ionic strength, on the extraction efficiency. The investigation of influences was focused on the application area of the SDME in the analysis of toxic organic compounds in samples of various origin, mainly in food and the environmental application area. 


\section{The single drop microextraction modes}

SDME, as a sample preparation technique, which was combined with gas chromatographic (GC) analysis, employing a simple and inexpensive apparatus involving negligible solvent consumption was described for the first time by Jeannot and Cantwell in 1996 (Jeannot and Cantwell, 1996). A small drop $(8 \mu \mathrm{L})$ of a water-immiscible organic solvent, containing an internal standard, was located at the end of a Teflon rod which was immersed in a stirred aqueous sample solution. After the solution was stirred for a prescribed period of time, the probe was withdrawn from the aqueous solution, and the portion of $1 \mu \mathrm{L}$ of the organic phase was sampled with a microsyringe and injected into the GC with flame ionization detection for quantification (Jeannot and Cantwell, 1996). However, the containment of the organic drop in a recess at the end of the Teflon rod was inconvenient because it required filling the recess with the drop of solvent at the start of the experiment and sampling the drop with a microsyringe prior to injection into the gas chromatograph at the end of the experiment (Jeannot and Cantwell, 1996).

To reduce the number of manipulation steps with extract, some improvements were performed by suspending a $1 \mu \mathrm{L}$ drop of organic solvent directly from the tip of a microsyringe needle immersed in the aqueous phase. The drop remained attached to the needle tip at stirring speeds up to $2000 \mathrm{rpm}$. Subsequently, the solute extracted into the $1 \mu \mathrm{L}$ drop was retracting back into the needle, withdrawing the needle from the aqueous solution, and injecting directly into the GC. The advantage of the $1 \mu \mathrm{L}$ system over the Teflon rod system was a faster rate of extraction (Jeannot and Cantwell, 1997).

Nowadays, seven different modes of single drop microextraction are presented. The base classification of these is dividing into two sub-categories, two and three-phase techniques, which exist in equilibrium. Two-phase techniques include:

- direct immersion SDME (DI-SDME),

- continuous flow microextraction (CFME),

- drop-to-drop microextraction (DDME),

- directly suspended droplet microextraction (DSDME),

and three-phase techniques include:

- headspace SDME (HS-SDME),

- liquid-liquid-liquid microextraction (LLLME),

- solvent-supported microextraction (SSME).

DI and HS are two the most common SDME modes occurred. In direct immersion SDME, the microdrop of an extraction solvent is in direct contact with an aqueous sample. The solvent must be immiscible with water, which involves the use of nonpolar or very slightly polar solvents. This mode is suitable for the extraction of nonpolar or moderately polar volatile and semivolatile analytes from relatively clean matrices, such as tap water. Removing any insoluble or particulate impurities from the sample is an important step for solvent drop stability during extraction. HS mode is usually used for the extraction and pre-concentration of the volatile compounds (Jeannot et al., 2010; Jain and Verma, 2011; Zhao et al., 2006). HS mode is based on reaching the equilibrium conditions between water sample, headspace area and the organic solvent drop placed on the tip of the needle. Volatile analytes are extracted from liquid or solid samples, also from the complex matrices containing solid particles (Kokosa, 2015).

CFME was reported for the first time by Liu and Lee in 2000. A polyetheretherketone (PEEK) tubing, commonly used in HPLC plumbing, was used as a holder of the extraction solvent drop. The extraction solvent was introduced through a conventional HPLC injection valve into a glass chamber by a microsyringe and held at the outlet tip of a PEEK connecting tube. The sample solution was flown right through the tube and the extraction glass unit to the waste. The solvent drop interacted continuously with the sample solution and the extraction was proceeded simultaneously. The undesirable air bubbles formation was avoided by the application of an HPLC injection valve, and a precise control of the size of the solvent drop was achieved (Liu and Lee, 2000). Over time, the conventional CFME method was modified, and a recycling-flow system in which the waste from the chamber was returned to the sample vial was developed (Xia et al., 2005). CFME is limited to extraction of nonpolar or slightly polar semivolatiles, such as selected pesticides, PAHs or aromatic compounds, due to the fact that only nonpolar extraction solvents are stable in the flowing system and the extent of their dissolution in the flowing sample is small. The other disadvantage of this mode is the necessity for additional equipment, such as a microinfusion pump (Płotka-Wasylka et al., 2016). For the complex biological samples, when the volume of the drop and the organic solvent is in microliter scale, DDME mode is available for extraction (Jeannot et al., 2010). DDME is a miniaturized version of DI-SDME. In this mode, the sample and organic solvent volumes are in microliter scale. Using small sample and solvent volumes is causing fast reaching equilibrium conditions between organic and water phase due to the high value of the kinetic constant. The enrichment factor is small, what brings the advantage of selectivity, which is provided by an extensive sample cleanup (Płotka-Wasylka et al., 
2016). Microliters of samples, such as blood, urine, and other fluids are used (Kokosa, 2015). In the DSDME, the microdrop of solvent is supplied to the surface of a stirred immiscible aqueous sample. The sample is stirred by a stirring bar placed on the bottom of the sample cell. After the extraction time, the microdrop of solvent is withdrawn by a syringe and analysed subsequently. Under the proper stirring conditions, the suspended droplet can easily remain in a top-center position of the aqueous sample (Yangchen et al., 2006). It means, that DSDME is based on a self-stable single microdrop system, avoiding use requirements for special equipment or other supporting materials. The challenge of keeping the drop on the tip of microsyringe needle in a vigorously stirred sample is obviated. The drop volume used for DSDME is relatively large $(>5 \mu \mathrm{L})$ and can be coupled with various analytical instruments easily (Mingyuan et al., 2009). Important is the size and shape of the stir bar, because it has a significant effect on the shape of the drop, which in turn can lead to difficulty in sampling. In addition, analyte adsorption on the surface of the stir bar was inevitable (Płotka-Wasylka et al., 2016).

The organic solvent for the LLLME must be water immiscible with the density lower than the density of water. The components are extracted from the sample solution into an organic solvent and simultaneously re-extracted from the organic solvent into the acceptor solution (a few microlitres) at a suitable $\mathrm{pH}$. The interface between two aqueous solutions is formed by the organic solvent. For acidic analytes, the $\mathrm{pH}$ of the sample solution is adjusted to lower values to suppress the ionization of the analytes for the extraction into the organic solvent. At the same time, the $\mathrm{pH}$ of the acceptor solution is maintained at a higher level to create ionization of the analytes. Thus, the analytes are converted to ions which are eliminated from the organic membrane and consequently accumulate in the acceptor solution (Płotka-Wasylka et al., 2016).

\section{Experimental parameters of SDME}

Several parameters such as the physical and the chemical character of analytes, properties and purity of extraction solvent, type of microsyringe, sample volume, temperature, ion strength and $\mathrm{pH}$ of sample affect efficiency of the extraction procedure. Furthermore, it is critical to find optimal extraction conditions which provide drop stability and high sensitivity. The case of dislodgment of the microdrop hanging from the tip of the microsyringe needle during the extraction process limits the use of extended extraction times, high stirring rates, increased sample temperature and the type of sample matrix to relatively clean (no solid particles present) (Carlos et al., 2013; Amde et al., 2015).

\section{Extraction solvent}

The selection of the extraction solvent is a critical step for SDME performance. The selected solvent must provide efficient and selective mass transfer of the analytes into the organic phase and preserve the stability of the microdrop during the extraction. A viscosity of the extraction solvent must be chosen adequate, for the stable formation and retention of the microdrop on the tip of the microsyringe needle during the extraction. Furthermore, high boiling point and low vapour pressure, in order to minimize any evaporation during the extraction process should be considered too. The principle "like dissolves like" is required, thus several water immiscible solvents differing in polarity should be tested. For DI-SDME the main requirement is the immiscibility of the solvent in the aqueous phase (Psillakis and Kalogerakis, 2002; Jeannot et al., 2010). Solvent purity is another important factor in SDME, especially for the analysis of the very dilute solutions. Impurities, such as xylene present in toluene, or oxidation products, such as aldehydes and alcohols in decane may interfere. Trace impurities can be useful, only in the case they may be used such as internal standards. Furthermore, drop integrity can be monitored using standard, which can also be added to the solvent. This is especially important when using an autosampler, to ensure that the drop is not lost during sampling. The standard can also be used to account for small sample-to-sample variations in drop size and solvent wicking on the needle (Jeannot, 2010).

Perreira and de Andrade tested three extraction solvents for the extraction of 19 pesticide residues from the coconut water, which is complex matrix consisting from sugars, salts, vitamins, minerals and amino acids. Toluene, cyclohexane and isooctane were studied as extraction solvents. It was observed, that all studied solvents provided satisfactory results. Toluene was selected due to the low toxicity and compatibility with the GC system. A drop volume of $1 \mu \mathrm{L}$ was chosen, showing a good drop stability and extraction efficiency of pesticides in order to ensure reproducibility and to allow the use of fast stirring (Perreira and de Andrade, 2014).

Dynamic microwave-assisted extraction (DMAE) coupled to SDME was investigated for the extraction of 7 organophosphorus pesticides from tea by Wu et al. in 2015. Pesticides were extracted by $25 \%$ (v/v) ethanol in water and purified with acidic alumina in the first stage of DMAE, and then pre-concentrated in the SDME stage by carbon tetrachloride. Carbon tetrachloride exhibited the highest extraction ef- 
ficiency for the majority of pesticides. An improved extraction chamber was established and used as a holder of the microdrop, and continuous flow of aqueous solution was passed through the microdrop by means of a micro-infusion pump. The microdrop was stable under employed conditions. After extraction, the microdrop was prepared for retraction into the microsyringe, and directly analysed by GC-MS without any filtration or clean-up step (Wu et al., 2015).

DMAE was employed in the combination with CFMA, to extend the application of the SDME to complex solid samples. DMAE-CFMA was used for the extraction of 8 organophosphorus pesticides from the vegetable. The parameters that affect the extraction efficiency, including the type and volume of the microdrop solvent were thoroughly investigated. The analytes were extracted from the vegetable sample, transferred from the extraction vessel to the chamber and then moved from extraction solvent into the microdrop. After extraction, the microdrop was retracted back into the microsyringe and introduced into GC-MS system for the analysis. Seven types of water-immiscible organic solvents, including $n$-hexane, cyclohexane, dichloromethane, chloroform, carbon tetrachloride, ethyl acetate, and toluene were tested. Dichloromethane and ethyl acetate showed significant losses in the continuous sample flow. Toluene exhibited the highest extraction efficiency for most of the pesticides and good stability of the drop. Therefore, toluene was selected for the following experiments. The volume of the microdrop from 1.5 to $3.5 \mu \mathrm{L}$ was studied, and it was found out that exceeded volume $3.5 \mu \mathrm{L}$, the drop became too unstable to suspend at the needle tip, thus $2.5 \mu \mathrm{L}$ was chosen for subsequent extractions (Wu et al., 2016).

SDME was used as a fast and simple extraction technique to pre-concentrate 18 organochlorine pesticides from water samples with a complex matrix. Solvent selectivity was evaluated, exposing $2 \mu \mathrm{L}$ of organic solvent drop immersed in a $10 \mathrm{~mL}$ deionized water sample, fortified with all target analytes at the appropriate concentration level. Toluene, $n$-hexane and isooctane were tested as a potential extraction solvent, and enrichment factors were calculated for each solvent. As a result, toluene provided optimal usage conditions, contrary, hexane presented dropstability problems (Cortada et al., 2009).

For the extraction of 12 hydroxylated metabolites of polycyclic aromatic hydrocarbons (PAHs) in seawater, the derivatization reagent was added to the extraction solvent in order to realize one-step extraction and derivatization. Seven different organic solvents including ethyl acetate, pentane, toluene, xylene, cyclohexane, hexane, and mixture of toluene:cyclo- hexane $(1: 1, \mathrm{v} / \mathrm{v})$ were investigated. More efficient extraction of the low molecular weight hydroxylated PAHs derivatives was achieved by using toluene as the extraction solvent, while for the extraction of high molecular weight hydroxylated PAHs derivatives cyclohexane was suitable as the extraction solvent. Therefore, toluene:cyclohexane $(1: 1, \mathrm{v} / \mathrm{v})$ was selected as a compromise for the following experiments (Wang et al., 2017).

Extraction of chlorobenzenes, belonging to a class of environmental pollutants usually used as industrial solvents, pesticides, dielectric fluids, deodorant and chemical intermediates, was studied by Vidal et al. in 2015. The solvent selectivity was evaluated after exposing $3 \mu \mathrm{L}$ organic solvent drop to the headspace of a $15 \mathrm{~mL}$ glass vial containing $10 \mathrm{~mL}$ deionised water samples. From the three tested solvents, $n$-hexane had the tendency to evaporate at faster rates once exposed to the air. $n$-Heptane was found to be more resistant to the evaporation due to its lower vapour pressure and resulted in enhanced extraction of target analytes when compared to $n$-hexane. Overall, toluene provided satisfactory results by combining the highest extraction efficiency as well as having the lowest vapour pressure (Vidal et al., 2005).

Amvrazi and Tsiropoulos were extracting pesticides from vegetable samples (Amvrazi and Tsiropoulos, 2009). Toluene and mixture of toluene: ethyl acetate $(9: 1, \mathrm{v} / \mathrm{v})$ were tested for extraction. Toluene showed the highest extraction efficiency for all pesticides except dimethoate. The organic drop volume was optimized by decreasing the drop volume from 2.0 to $1.6 \mu \mathrm{L}$ and $90 \%$ of the drop volume was withdrawn into the microsyringe. The occasional uplift of the drop lengthwise the needle of the syringe at the time the drop was exposed in the stirred solution was observed by using $2 \mu \mathrm{L}$ volume of the drop. Hence, a volume of $1.6 \mu \mathrm{L}$ of toluene was chosen in further work (Amvrazi and Tsiropoulos, 2009).

For the extraction of pesticides from a wine sample, several solvents with different polarity and water solubility were studied to achieve satisfactory extraction recoveries. Toluene, $n$-hexane, chloroform and isooctane were thoroughly investigated. Isooctane was selected, due to its properties such as higher immiscibility with water compared to other solvents, high boiling point and larger areas of the target analyte peaks obtained after GC-MS analysis. The sensitivity of the proposed SDME method was studied by experiments performed with a drop volume in the scale $0.5-3 \mu \mathrm{L}$. It was shown, that drop greater than $2 \mu \mathrm{L}$ was unstable on the needle tip and difficult to handle. Therefore, $2 \mu \mathrm{L}$ of isooctane was chosen as the optimal volume of extraction solvent (Garbi et al., 2010). 


\section{Agitation}

Sample agitation is an important factor for the reduction of extraction time. From the film theory of convective-diffusive mass transfer, the agitation of the sample solution enhances the extraction efficiency and reduce the extraction time, since the thickness of the stagnant film around the extracting phase (i.e. Nernst diffusion film) decreases with increasing stirring rate, and it results in faster extraction rate (Pena-Pereira et al., 2010). Magnetic stirring, mechanical vibration or syringe plunger motion are usually used for the sample agitation to increase the amount of convective mixing or interfacial contact area. The time required to reach equilibrium in SDME depends on the type and degree of agitation, phase volumes, interfacial contact area and equilibrium distribution constant (Jeannot and Cantwell, 1996; Jeannot et al, 2010).

Stirring was the factor that determined a significant increase in the peak area of pesticides for the extraction of pesticides from coconut water. Better extraction efficiency was obtained when using rapid stirring. Higher extraction efficiencies of pesticides for the stirring rates 200 and $300 \mathrm{rpm}$ were observed. The rate of $200 \mathrm{rpm}$ was chosen because a smaller solution disturbance was occurred compared to high speed of stirring. Greater microdrop stability at the tip of the microsyringe needle was observed for the low stirring rate (Perreira dos Anjos and de Andrade, 2014). The same conditions were applied for the extraction of pesticides from wine by Perreira dos Anjos and de Andrade in 2015 (Perreira dos Anjos and de Andrade, 2015). Stirring rate of magnetic stirring, $380 \mathrm{rpm}$, was selected for the extraction of pesticides from water samples (Cortada et al., 2009). For the extraction of hydroxylated PAHs in sea waters, the highest abundance was obtained when the stirring rate reached $350 \mathrm{rpm}$, and the abundance decreased for higher agitation speed due to the unstable drop and loss of the extraction solvent (Wang et al., 2017). In the case of HS-SDME, stirring the aqueous sample resulted in a degree of convection of the headspace. Increasing the speed of sample agitation, the results revealed that agitation dramatically enhanced extraction efficiency reaching a maximum at $1000 \mathrm{rpm}$. At $1250 \mathrm{rpm}$ (maximum speed of the magnetic stirrer), the stability of the drop was affected, depending on the analyte, the resulting analytical signal either decreased or remained the same (in comparison to $1000 \mathrm{rpm}$ ) (Vidal et al., 2005).

Evaluation of the optimum agitation rate for pesticides extraction from vegetables was performed at different stirring rates $(150,250$ and $350 \mathrm{rpm})$. It was observed, that the relative peak areas of almost all target analytes (except for pyriproxyfen) was increased with the increase of stirring rate. Since higher than $350 \mathrm{rpm}$ stirring rates were found to increase the incidence of drop damage and/or dissolution, the optimum stirring rate was set at $350 \mathrm{rpm}$ (Amrvazi and Tsiropoulos, 2009).

\section{Temperature}

Raising the temperature for HS-SDME can accelerate the rate of mass transfer of analytes from the sample to the headspace and to increase the amount of analytes transferred to the headspace. Higher temperatures lead to the decrease of the organic solvent-headspace distribution constant, resulting in the low sensitivity of the determination. The loss of sensitivity can be avoided when the extracting solvent is cooled while the sample is heated. This strategy significantly complicates the experimental setup. Thus, it should be used only for ultra-trace analyses or for highly volatile analytes with low solvent-headspace distribution constants (Jeannot et al., 2010).

Influence of temperature was deeply studied for the HS-SDME extraction of five organophosphorus pesticides in soil. In this case, increasing the extraction temperature showed two different effects. The positive effect on the extraction yield, through enhancing the analyte transfer to the water and consequently to the headspace as well as enhancing diffusion rate through the water and headspace was observed. The negative effect on the extraction efficiency was observed by increasing the evaporation of extraction solvent and decreasing the partitioning of the analytes into the solvent drop. The effect of the extraction temperature for all analytes, as well as the overall response was positive, this factor was set at its high level $\left(60{ }^{\circ} \mathrm{C}\right)$ to obtain the best results (Salemi et al., 2013).

In the case of alcohol content samples, such as wine sample, experiments at temperatures between 20-35 ${ }^{\circ} \mathrm{C}$ were performed. The presence of ethanol in the samples using a temperature of more than $35{ }^{\circ} \mathrm{C}$ resulted into the instability of the drop held on the tip of the microsyringe, while intense bubble formation in the bulk solution caused its final dislodgment. Experiments carried out at temperatures between $20^{\circ} \mathrm{C}$ and $35^{\circ} \mathrm{C}$ for 10 minutes extraction period showed optimal extraction efficiency of analytes and good reproducibility (Garbi et al., 2010).

\section{Salt addition and $p H$}

The $\mathrm{pH}$ of the sample solution is one of the most important factors affecting the SDME. It can significantly influence the form of the analytes in the aqueous phase and consequently its solubility and extractability. SDME is an equilibrium-based 
extraction approach, and the increase of the ionic strength of the aqueous solution could affect the analyte transfer (Wang et al., 2017).

A positive effect of salt addition has been reported for HS-SDME of 5 organophosphate pesticides. Several amounts of sodium chloride were used to decrease the solubility of the analytes in the aqueous part and to enhance the extraction efficiency. A significantly positive effect was observed for only thionazin. Application of saturated salt solution for salting out is not appropriate, because it may contain undissolved particles that can cause drop degradation, especially in DI-SDME mode (Salemi et al., 2013).

DMAE in combination with CFME system was optimized and validated for extraction of eight organophosphorus pesticides in vegetables by $\mathrm{Wu}$ et al. in 2016. The method extended the application of the single drop microextraction to complex solid samples. Extraction, separation, and enrichment were performed in a single step, which could greatly simplify the operation and reduce the whole pretreatment time. No filtration and clean-up process were used. Generally, $\mathrm{NaCl}$ was used to adjust the ionic strength, which can improve the analyte partition between the aqueous phase and the organic phase. However, in the traditional CFME, the opposite conclusion was drawn, possibly because the $\mathrm{NaCl}$ dissolved in the aqueous solution may change the physical properties of the Nernst diffusion film and reduce the rate of diffusion of the target analyte into the drop (Psillakis and Kalogerakis, 2001). Addition of different amounts of $\mathrm{NaCl}(0-10 \%$, w/v) was tested. The experimental results indicated that the recoveries of the analytes first increase slightly, then decrease, and achieve a maximum at $3 \% \mathrm{NaCl}$ (Wu et al., 2016). Wang et al. investigated the ionic strength and $\mathrm{pH}$ of the extraction of hydroxylated PAHs. Different concentrations of $\mathrm{NaCl}(0 \%, 5 \%$, $15 \%$, and $30 \%$ ) were used. The abundance of hydroxylated PAHs increased with the increasing of ionic strength, and the water solubility of target compounds was decreasing. The highest abundance of most target compounds was achieved for $30 \%$ of $\mathrm{NaCl}$ added. The effect of $\mathrm{pH}$ of the solutions on the extraction efficiency was studied by adjusting $\mathrm{pH}$ from 2 to 7 . It was found that most of hydroxylated PAHs reached their highest abundance when $\mathrm{pH}$ was adjusted to 2 (Wang et al., 2017). The negative effect of the higher ionic strength was observed for the extraction of organochlorine pesticides in water samples with complex matrices, reaching a maximum at $2.6 \%(\mathrm{w} / \mathrm{v})$ of $\mathrm{NaCl}$. Apart from the salting-out effect, the presence of salt was assumed to cause a secondary effect and change the physical properties of the extraction film, thus reducing the diffusion rates of the analytes toward the drop. Since this variable had a non-significant effect and the presence of salt also caused drop instability, the following extractions were performed without $\mathrm{NaCl}$ addition (Cortada et al., 2009).

SDME was successfully applied for the extraction of atrazine and its major degradation products from environmental waters (Yohannes et al, 2016). The effect of salt concentration on the extraction of the analytes by adding different quantities of $\mathrm{NaCl}$ varied from 2.5 to $15 \%(\mathrm{w} / \mathrm{v})$ was investigated. The results demonstrated an initial increase in extraction efficiency, with increased salt concentration, the maximum being reached at $7.5 \%(\mathrm{w} / \mathrm{v})$, followed by a decrease in the extraction efficiency with further increase in the salt concentration (10-15\%). Extractability of the weak organic bases such as atrazine and its metabolites significantly depends on $\mathrm{pH}$ of the sample solution. In this case it was resulted, that the enrichment factor of the considered analytes exhibited increasing tendency with $\mathrm{pH}$ ranges of 2-4 and then the increase was noticed to be gradual up to $\mathrm{pH}$ 7.0. However, after the neutral $\mathrm{pH}$, the enrichment factor started declining. Therefore, $\mathrm{pH} 7.0$, where all the target compounds are deionized for efficient transfer into the extraction solvent, was chosen as the optimum sample solution $\mathrm{pH}$. Neutral $\mathrm{pH}$ has also been noted as adequate, for SDME extraction of cyanazine, simazine and atrazine by Ye et al. in 2007 (Yohannes et al., 2016; Ye et al., 2007). A small bell-mouthed extraction device for single-drop microextraction (SDME) is a new approach developed by Ye et al. (Ye et al., 2007). Analytical sensitivity was improved and stability of drop was markedly increased by increasing the suspended acceptor volume, contact area and the inner surface of the extraction device. The merits and advantages of the proposed method were demonstrated by using 1-octanol as an extractant and cyanazine, simazine and atrazine as model compounds were extracted. Extraction parameters were investigated, with the scope on $\mathrm{NaCl}$ addition and $\mathrm{pH}$. NaCl concentration was ranging from 0 to $25 \%$. It was resulted in the initial increase in the extraction efficiency with an increase in salt concentration, with a maximum at $15 \%$, followed by a decrease in extraction efficiency with further increase in salt concentration. A $15 \%(w / v) ~ N a C l$ addition was selected since it provided the best extraction efficiency for all the analytes. Furthermore, a wide range of sample $\mathrm{pH}$ from 3 to 11 was evaluated. Based on the results, it was evident that the extraction efficiency at $\mathrm{pH}<5$ or $\mathrm{pH}>9$ were lower than that at the $\mathrm{pH}$ range of 5-9 for each analyte. This may have resulted from the hydrolysis of triazines under strongly acidic or basic aqueous environ- 
ments and the fact that they are relatively stable under neutral conditions. The experimental results indicated that the highest extraction efficiency was achieved in neutral conditions (Ye et al., 2007).

\section{Conclusion}

After the introduction of SDME in 1996, SDME has undergone many technical changes and it is currently known in various modes. The efficiency of SDME and solvent drop stability is directly depending on the relevant extraction parameters, hence selected extraction parameters such as extraction solvent and its volume, agitation, temperature, $\mathrm{pH}$ of the sample and ionic strength on the extraction were discussed and demonstrated by examples of particular application areas. Is has been noted, that the water-immiscible solvents differing in polarity are commonly used for the extraction of toxic organic compounds by SDME, particularly toluene, isooctane, cyclohexane, carbon tetrachloride, dichloromethane, chloroform, ethyl acetate, $n$ - hexane, pentane, xylene and specific mixtures in various ratios. It was shown, that toluene and carbon tetrachloride are suitable for the extraction of pesticides from complex matrix without causing instability of the microdrop and losing the analytes. The stirring of the sample is provided by various techniques and, in general, high stirring rates, for instance 1000-1300 rpm, are applied for HSSDME, and low rates ranging between 200 and $400 \mathrm{rpm}$ are mostly applied for DI-SDME. Special attention to the selection of temperature should be given. The increase of temperature influences not only the transfer of analytes into the extraction phase, but it can cause particular drawbacks, e.g. bubble formation in the bulk solution. From the other parameters, $\mathrm{pH}$ and salt addition significantly affect in some cases the extraction efficiency of various modes of SDME. Salt addition had a strong influence on the extraction of hydroxylated PAHs, as well as the acid $\mathrm{pH}$ was required. On the other hand, for the extraction of selected pesticides, the neutral $\mathrm{pH}$, the absence of salt addition or lower salt addition was optimal. It is necessary optimize this parameter in dependence on the chemical nature of individual extracted analytes.

Miniaturized extraction methods such as SDME represent a new approach that is currently receiving a great deal of interest of researchers in the area of sample preparation methods development.

\section{Acknowledgement}

This work was supported by the Slovak Research and Development Agency under the contract No. SK-SRB2016-0006. SZ would like to acknowledge the support by the STU Grant scheme for Support of Young Researchers and by the STU Grant scheme for Support of Excellent Teams of Young Researchers.

\section{References}

Andraščíková M, Matisová E, Hrouzková S Separation and Purification Reviews 44: 1-18.

Andruch V, Kocúrová L, Balogh IS, Škrlíková J (2012) Microchemical Journal 102: 1-10.

Amde M, Tan ZQ, Liu R, Liu JF (2015) Journal of Chromatography A 1395: 17-15.

Amvrazi EG, Tsiropoulos NG (2009) Journal of Chromatography A 1216: 2789-2797.

Asensio-Ramos M, Ravelo-Pérez LM, GonzálezCurbelo MÁ, Hernández-Borges J (2011) Journal of Chromatography A 1218: 7415-7437.

Carlos EA, Alves RD, de Queiroz MERL, Neves AA (2013) Journal of Brazilian Chemical Society 24: 1217-1227.

Cortada C, Vidal L, Tejada S, Romo A (2009) Analytica Chimica Acta 638: 29-35.

Garbi A, Sakkas V, Fiamegos YC, Stalikas CD, Albanis T (2010) Talanta 82: 1286-1291.

Hrouzková S (2017) In: "Food analysis: Innovative analytical tools for safety and quality assessment", Umile Gianfranco Spizzirri, Giuseppe Cirillo (Ed), John Wiley \&Sons, Hoboken, New Jersey and Scrivener Publishing, Beverly, MA, 2017, p. 221.

Hrouzková S, Szarka A, Zichová S (2018) Chemické listy 112: $165-174$.

Jain A, Verma KK (2011) Analytica Chimica Acta 706: $37-65$

Jeannot MA, Cantwell FF (1996) Analytical Chemistry 69: 2236-2240.

Jeannot MA, Cantwell FF (1997) Analytical Chemistry 69: 235-239.

Jeannot MA, Przyjazny A, Kokosa JM (2010) Journal of Chromatography A 1217: 2326-2336.

Kokosa JM (2015) Trends in Analytical Chemistry 71: 194-204.

Liu W, Lee HK (2000) Analitical Chemistry 72: 44624467.

Mingyuan G, Yangcheng L, Guangsheng L (2009) Analytica Chimica Acta 648: 123-127.

Pena-Pereira F, Lavilla I, Bendicho C (2010) Trends in Analytical Chemistry 29: 617-628.

Perreira dos Anjos J, de Andrade JB (2014) Microchemical Journal 112: 119-126.

Perreira dos Anjos J, de Andrade JB (2015) Microchemical Journal 120: 69-76.

Płotka-Wasylka J, Owczarek K, Namieśnik J (2016) Trends in Analytical Chemistry 85: 46-64.

Psillakis E, Kalogerakis N (2001) Journal of Chromatography A 938: 113-120.

Psillakis E, Kalogerakis N (2002) Trends in Analytical Chemistry 2: 53-63.

Salemi A, Rasoolzadeh R, Nejad MM, Vosough M (2013) Analytica Chimica Acta 769: 121-126.

Sarafraz-Yazdi A, Amiri A (2010) Trends in Analytical Chemistry 29: 1-14.

Tobiszewski M, Mechlinska A, Zygmunt B, Namiesnik J (2009) Trends in Analytical Chemistry 28: 943-951. 
Vidal L, Canals A, Kalogerakis N, Psillakis E (2005) Journal of Chromatography A 1089: 25-30.

Wang X, Ying L, Cai F, Qing Q, Yuan K, Chen B, Luan T (2017) Talanta 164: 727-734.

Wu L, Hu M, Li Z, Song Y, Cui Y, Zhang H, Yu A, Ma Q, Wang Z (2016) Food Chemistry 192: 596-602.

Wu L, Hu M, Li Z, Song Y, Zhang H, Yu A, Ma Q, Wang Z (2015) Journal of Chromatography A 1407: 42-51.

Yangcheng L, Quan L, Guangsheng L, Youyuan D (2006) Analytica Chimica Acta 566: 259-264.

Ye C, Zhou Q, Wang X (2007) Journal of Chromatography A 1139: 7-13.
Yohannes A, Tolesa T, Merdassa Y, Megersa (2016) Journal of Analytical and Bioanaltical Technology 7: $1-8$.

Xia L, Hu B, Jiang Z, Wu Y, Li L, Chen R (2005) Journal of Analytical Atomic Spectrometry 20: 441-446.

$\mathrm{Xu}$ L, Basheer Ch, Lee HK (2007) Journal of Chromatography A 1152: 184-192.

Zhao E, an L, Jiang S, Wang Q, Zhou Z (2006) Journal of Chromatography A 1114: 269-273. 